\title{
Keeping tetramers together
}

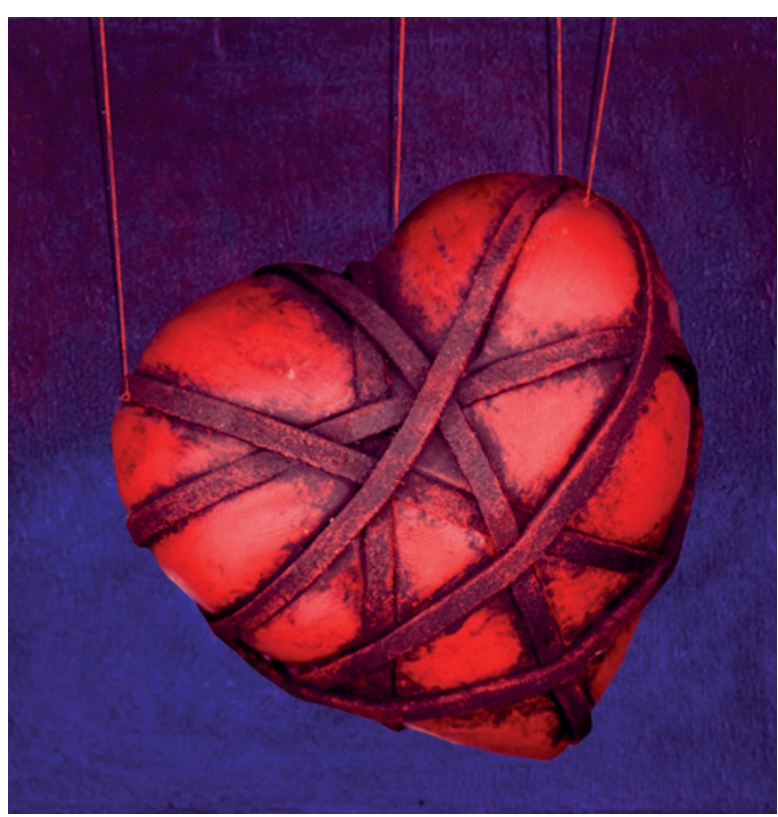

Transthyretin (TTR) is a tetrameric protein that transports thyroxine and retinol in the blood; however, it is also amyloidogenic as it can form disease-causing aggregates. In the heart, aggregates of TTR lead to amyloid cardiomyopathy for which there is currently no approved therapy. A new study has identified several drug candidates that inhibit the first step towards amyloid formation by preventing the dissociation of TTR tetramers.

Several TTR stabilizers identified by rational design are in clinical trials, but their resemblance to non-steroidal anti-inflammatory drugs (NSAIDs) is likely to lead to cardiovascular problems and preclude their long-term use in patients with cardiac amyloidosis. In Science Translational Medicine, Graef and colleagues describe the development of a fluorescence polarization-based high-throughput screen to detect small molecules that bind to the thyroxine-binding pocket of TTR under physiological conditions. Over 130,000 compounds were screened, and the ones with the highest binding affinity (in the nanomolar range) were then assessed for their TTR-stabilizing activity and effect on cyclooxygenase 1 activity to avoid the off-target toxicity issues associated with NSAIDs.

Analyses of the crystal structures of the four best TTR stabilizers that did not affect cyclooxygenase 1 activity revealed that despite their distinct ring structures they are all able to form a combination of electrostatic and hydrophobic interactions with adjacent TTR subunits that help to stabilize the dimer-dimer interface and increase the activation energy for tetramer dissociation.

Owing to the lack of animal models of cardiac amyloidosis, the authors next examined the effects of these compounds in cardiomyocytes treated with the most common cardiomyopathy-associated TTR mutant: V122I-TTR. They found that the compounds rescued cells from TTR proteotoxicity without causing cytotoxicity. Furthermore, these compounds seemed to be selective for TTR as they were able to stabilize TTR tetramers in the presence of all human serum proteins. These compounds represent exciting new leads for the development of novel TTR stabilizers that could lead to the development of more effective treatments for cardiac amyloidosis and potentially other TTR-associated amyloidoses such as senile systemic amyloidosis and familial amyloid polyneuropathy.

Monica Hoyos Flight

ORIGINAL RESEARCH PAPER Alhamadsheh, M. M. et al. Potent kinetic stabilizers that prevent transthyretin-mediated cardiomyocyte proteotoxicity. Sci. Transl. Med. 3, 97 ra81 (2011) 